\section{Qualidade da notificação de anomalias congênitas pelo Sistema de Informações sobre Nascidos Vivos (SINASC): estudo comparativo nos anos 2004 e 2007}

\author{
Quality of birth defect reporting in the Brazilian \\ Information System on Live Births (SINASC): \\ a comparative study of 2004 and 2007
}

\author{
${ }^{1}$ Craniofacial Division, \\ University of Washington. \\ Seattle, USA. \\ 2 Escola Nacional de Saúde \\ Pública Sergio Arouca, \\ Fundação Oswaldo Cruz, Rio \\ de Janeiro, Brasil. \\ Correspondência \\ D. V. Luquetti \\ Craniofacial Division, \\ University of Washington. \\ 4735 Ravenna Ave NE apt 1 , \\ Seattle, WA 98105, USA. \\ luquetti@uw.edu
}

\begin{abstract}
This study compared the validity of birth defect cases reported in the Brazilian Information System on Live Births in 2004 and 2007 in seven municipalities, out of a total of 27,945 live births in 2004 and 25,905 in 2007. The study also describes quality improvement measures in this area. The Latin American Collaborative Study on Congenital Malformations was used as the gold standard for analyzing the validity of birth defect diagnoses. In 2004, at least $40 \%$ of birth defects went unreported, and this situation persisted in 2007. Only one hospital showed a significant increase in reporting sensitivity, from $56.9 \%$ to $96.8 \%$. Sensitivity decreased significantly in two hospitals (from $62.7 \%$ to $41.75 \%$ and from $66.5 \%$ to $32.2 \%$, respectively). The positive and negative predictive values and specificity remained above $80 \%$. Only two municipal health departments and four hospitals implemented measures to improve reporting of birth defects. The results indicate the need for investments in the quality of birth defect information on birth certificates.
\end{abstract}

Congenital Abnormalities; Birth Certificates; Information Systems
Daniela Varela Luquetti 1,2 Rosalina Jorge Koifman ${ }^{2}$

\section{Introdução}

O objetivo de avaliar a qualidade de dados dos sistemas de informação é fornecer elementos sobre a acurácia das medidas e indicadores gerados por esses sistemas. A avaliação da completude, validade e confiabilidade deve ser realizada periodicamente, para assegurar aos gestores da saúde que as decisões tomadas estão baseadas em informação de qualidade 1 .

O Sistema de Informações sobre Nascidos Vivos (SINASC) foi implementado em 1990 e, a partir de 1999, foi acrescentado um novo campo (campo 34), que possibilita a notificação das anomalias congênitas. Até o ano de 2006, apesar de existir espaço na Declaração de Nascido Vivo para a descrição detalhada das anomalias congênitas, apenas um código de anomalia podia ser digitado na informatização do banco de dados. A partir de então, todos os diagnósticos de anomalias congênitas descritas puderam ser codificados e digitados no SINASC. Este sistema de informação possui características únicas, das quais poucos países no mundo dispõem, tais como: cobertura nacional, sistema eletrônico de envio de dados e disponibilidade de informação desagregada.

A partir da adição do campo 34 na Declaração de Nascido Vivo, o Brasil passou a dispor de um sistema de informação que tornou possível a implementação de um programa nacional de vigilância epidemiológica de anomalias congê- 
nitas, com o objetivo de monitorar a prevalência dessas condições, conduzir estudos de fatores de risco, monitorar exposição a teratógenos e planejar programas de prevenção e tratamento de anomalias congênitas. No entanto, a qualidade dos dados sobre estas não foi avaliada de maneira abrangente até agora, já que as informações geradas ainda apresentam limitações para seu uso. Alguns estudos avaliaram o grau de completitude em determinados municípios ou hospitais $2,3,4,5,6$, e os resultados mostraram uma melhora no grau de preenchimento nas pesquisas mais recentes. A avaliação da confiabilidade foi realizada no Município do Rio de Janeiro, comparando-se a informação sobre anomalias congênitas na Declaração de Nascido Vivo com o prontuário médico. Verificou-se ampla variabilidade do índice kappa ajustado pela prevalência, com melhores resultados nos aparelhos osteomuscular, geniturinário, digestivo e nas anomalias cromossômicas; os piores foram verificados nos sistemas nervoso central e cárdio-circulatório e nas malformações craniofaciais 7 . Em relação à validade dos diagnósticos de anomalias congênitas, observou-se, em uma maternidade de Ribeirão Preto, São Paulo, que mais de $60 \%$ dos casos com diagnóstico descrito no prontuário não foram notificados na Declaração de Nascido Vivo 5 . Na comparação com o Sistema de Informações sobre Mortalidade (SIM), no Município de São Paulo 2, foi observada subnotificação de $12 \%$, no entanto as prevalências observadas (menos que $0,8 \%$ ) indicam uma provável subnotificação em ambos os sistemas de informação.

Este estudo faz parte de um projeto de avaliação da qualidade das informações do SINASC no que se refere às anomalias congênitas em hospitais distribuídos nas regiões Nordeste, Sudeste e Sul do Brasil. Em uma primeira etapa, avaliamos a validade do diagnóstico de anomalia congênita e a confiabilidade da codificação em oito hospitais localizados em sete municípios para o ano de 2004 8,9. Esta segunda etapa do projeto teve como objetivo avaliar a evolução da validade do diagnóstico das anomalias congênitas no SINASC por meio da comparação entre os anos de 2004 e 2007, utilizando um registro de anomalias congênitas de base hospitalar como padrão. Além disso, foi investigado se os hospitais selecionados, as respectivas secretarias municipais de saúde (SMS) e/ou o Ministério da Saúde desenvolveram ações específicas para o aprimoramento da qualidade da informação do campo 34 da Declaração de Nascido Vivo.

\section{Material e métodos}

Foram utilizadas as informações contidas no banco de dados do SINASC que se referiam aos nascidos vivos, por ocorrência, nos anos 2004 e 2007, em oito hospitais dos municípios de João Pessoa (Paraíba), Salvador (Bahia), São Paulo e Campinas (São Paulo), Belo Horizonte (Minas Gerais) e Florianópolis e Joinville (Santa Catarina), num total de 27.945 nascidos vivos em 2004 e 25.905 em 2007.

Para a avaliação da notificação de recém-nascidos com anomalias congênitas pelo SINASC, utilizaram-se as informações contidas no banco de dados do Estudo Colaborativo Latino-Americano de Malformações Congênitas (ECLAMC) nos mesmos hospitais e anos. Criado em 1967, o ECLAMC visa ao registro de anomalias congênitas em nascidos vivos ou natimortos e à promoção de políticas de saúde para o estabelecimento de medidas preventivas dessas anomalias do desenvolvimento. Trata-se de um estudo epidemiológico de base hospitalar, com uma rede, em 2004, de cem hospitais, 16 destes no Brasil, cobrindo aproximadamente $1 \%$ dos nascimentos do país. A participação na rede ECLAMC é voluntária; profissionais interessados em participar entram em contato com a coordenação, com sede na Fundação Oswaldo Cruz (Fiocruz), no Rio de Janeiro. A busca de casos é ativa, isto é, todos os recém-nascidos de um hospital participante são examinados por um profissional treinado. Além do exame físico, uma ficha com cinquenta variáveis de possíveis fatores de risco é preenchida através de entrevista com a mãe durante o puerpério. Todas as anomalias maiores e menores diagnosticadas ao nascimento, em nascidos-vivos ou óbitos fetais pesando $500 \mathrm{~g}$ ou mais, são descritas de acordo com um manual de procedimentos e documentadas com fotos e radiografias sempre que possível. Todas as anomalias são codificadas em nível central, por meio de um código próprio de seis dígitos 10. Esse sistema, na ausência de outros específicos, cumpre o papel de única fonte de informação para a vigilância epidemiológica dessa morbidade e representa uma base de dados epidemiológicos de qualidade. Por esses motivos, o ECLAMC foi adotado como padrão-ouro para avaliar a validade de critério dos diagnósticos de anomalias congênitas no SINASC. Considerando que o SINASC somente registra nascidos vivos, para este estudo foram excluídos todos os casos do ECLAMC em óbitos fetais.

Os critérios utilizados para a seleção dos hospitais foram participação na rede ECLAMC de janeiro a dezembro de 2004 e número de nascidos vivos maior que mil por ano (hospitais com 
menos que mil nascidos vivos ao ano teriam em torno de 20 a 30 casos de anomalias congênitas, levando a instabilidade de proporções). Dos 16 hospitais participantes do ECLAMC em 2004, três foram excluídos porque intervenções para a melhoria da notificação de anomalias congênitas pela Declaração de Nascido Vivo já vinham sendo conduzidas desde 2001. Cinco outros hospitais foram excluídos por apresentarem menos de mil nascidos vivos ao ano. Os oito hospitais selecionados são públicos e realizam variadas atividades de ensino; eles foram renomeados HOSP1 a HOSP8, em ordem decrescente do total de nascimentos.

Para garantir a independência das informações obtidas nos dois sistemas, as equipes responsáveis pelo ECLAMC e SINASC, dentro de um mesmo hospital, não compartilhavam de informações ao longo do processo de caracterização das anomalias congênitas observadas. No entanto, a partir de 2006, a SMS de Campinas passou a utilizar o banco de dados ECLAMC do hospital deste município (HOSP4) como fonte de dados para complementar o banco de dados SINASC em relação às anomalias congênitas.

O banco de dados do SINASC contendo todos os nascidos-vivos foi vinculado ao banco de dados do ECLAMC. A vinculação não foi possível em 11 casos existentes no banco de dados do ECLAMC por inexistirem na base de dados do SINASC, sendo excluídos da análise. As variáveis utilizadas para a vinculação dos bancos de dados foram nome da mãe, peso ao nascimento e idade materna. Caso notificado foi definido como todo recém-nascido com o campo de anomalia congênita codificada como "sim" pelo SINASC. Os casos com anomalias menores, de pouca importância clínica, tais como nevos, orelhas displásicas e fístulas pré-auriculares, foram igualmente excluídos da análise. Um dos hospitais, HOSP8, não participou do ECLAMC em 2007 e, portanto, foi excluído da análise da comparação da validade de critério de diagnóstico.

As prevalências pelo ECLAMC e SINASC em 2004 e 2007, para cada hospital, foram avaliadas pelo intervalo de 95\% de confiança (IC95\%) da diferença das proporções.

Sensibilidade, especificidade, valores preditivos positivo e negativo foram calculados para o conjunto de casos de anomalias congênitas obtidos por meio da vinculação dos bancos de dados do SINASC e ECLAMC. Sensibilidade foi definida como a proporção de casos de anomalias congênitas detectados pelo ECLAMC, os quais foram notificados pelo SINASC; valor preditivo positivo, como a proporção de casos identificados pelo SINASC e também pelo ECLAMC como casos de anomalias congênitas; especificidade, como a proporção de casos que o ECLAMC notificou como não tendo anomalia congênita, os quais também foram notificados pelo SINASC como não tendo essa anomalia; valor preditivo negativo, como a proporção de casos nos quais o SINASC indicava não haver anomalia congênita, os quais também foram identificados dessa forma pelo ECLAMC. A validade de critério do SINASC para o diagnóstico de anomalias congênitas foi comparada, para cada hospital, para os anos $2004 \mathrm{e}$ 2007, pelos IC95\%.

Para a descrição das ações específicas realizadas em relação à notificação das anomalias congênitas pelo SINASC, um correio eletrônico foi enviado para os responsáveis pelo ECLAMC nos hospitais selecionados e para os responsáveis pelo SINASC nas respectivas SMS. O responsável pelo SINASC no Ministério da Saúde também foi contatado. O correio eletrônico indagava sobre qualquer ação (mudanças no preenchimento ou fluxo de informação, reuniões, cursos de capacitação, portarias, elaboração de manuais) realizada, no período de janeiro de 2005 a dezembro de 2007, pelos hospitais, pelas SMS ou pelo Ministério da Saúde.

\section{Resultados}

Nos hospitais avaliados, foi observada uma variação da prevalência das anomalias congênitas entre 2004 e 2007, segundo o ECLAMC. No entanto, somente em dois hospitais essas mudanças foram significativas: no HOSP1, de 4,3\% para 5,6\% (IC95\%: 0,005-0,021), e no HOSP7, de 3\% para 1,6\% (IC95\%: 0,002-0,025). Pelo SINASC houve diminuição significativa da prevalência de anomalias congênitas no HOSP5, de 5,3\% para 2,7\% (IC95\%: 0,015-0,037), e aumento nos HOSP4, de 3,2\% para 4,5\% (IC95\%: 0,003-0,024), e HOSP8, de $0,9 \%$ para $4,3 \%$ (IC95\%: 0,020-0,048) (Tabela 1).

Dos sete hospitais em que foi calculada a validade de critério, observou-se aumento na sensibilidade nos HOSP2, HOSP3, HOSP4 e HOSP6, entre 2004 e 2007, sendo este aumento significativo somente no HOSP4, de 56,9 (IC95\%: 49,1-64,4) para 96,8 (IC95\%: 92,6-99,0). Nos HOSP1, HOSP5 e HOSP7, houve diminuição na sensibilidade, sendo esta significativa somente para os HOSP1, de 62,7 (IC95\%: 49,1-64,4) para 41,7 (IC95\%: 49,164,4) e HOSP5, de 66,5 (IC95\%: 60,0-72,6) para 32,2 (IC95\%: 26,2-38,2). Os valores preditivos positivo e negativo e especificidade permaneceram com valores semelhantes para todas as unidades (Tabela 2).

Todos os gestores do SINASC e profissionais dos hospitais selecionados responderam ao 
Distribuição dos casos de anomalias congênitas no Sistema de Informações sobre Nascidos Vivos (SINASC) e no Estudo

Colaborativo Latino-Americano de Malformações Congênitas (ECLAMC), por hospital, em 2004 e 2007.

\begin{tabular}{|c|c|c|c|c|c|c|c|c|}
\hline & \multirow{3}{*}{$\begin{array}{c}\text { Ação de } \\
\text { aprimoramento } \\
\text { (campo 34) }\end{array}$} & \multirow[t]{3}{*}{ Nascidos-vivos } & \multicolumn{6}{|c|}{ Casos } \\
\hline & & & \multicolumn{2}{|c|}{ ECLAMC } & \multicolumn{2}{|c|}{ SINASC } & \multicolumn{2}{|c|}{ Subnotificados } \\
\hline & & & $\mathbf{n}$ & $\%$ & $\mathrm{n}$ & $\%$ & $\mathrm{n}$ & $\%$ \\
\hline \multicolumn{9}{|l|}{ HOSP1 } \\
\hline 2004 & Não & 7.138 & 306 & 4,3 & 192 & 2,7 & 114 & 37,2 \\
\hline 2007 & & 5.942 & 333 & 5,6 & 139 & 2,3 & 194 & 58,2 \\
\hline \multicolumn{9}{|l|}{ HOSP2 } \\
\hline 2004 & Não & 6.126 & 101 & 1,7 & 51 & 0,8 & 50 & 49,5 \\
\hline 2007 & & 5.646 & 103 & 1,9 & 58 & 1,0 & 45 & 43,7 \\
\hline \multicolumn{9}{|l|}{ HOSP3 } \\
\hline 2004 & Não & 3.900 & 125 & 3,2 & 67 & 1,7 & 58 & 46,4 \\
\hline 2007 & & 3.727 & 92 & 2,5 & 52 & 1,4 & 40 & 43,5 \\
\hline \multicolumn{9}{|l|}{ HOSP5 } \\
\hline 2004 & Não & 2.682 & 215 & 8,1 & 143 & 5,3 & 72 & 33,5 \\
\hline 2007 & & 2.818 & 236 & 8,4 & 76 & 2,7 & 160 & 67,8 \\
\hline \multicolumn{9}{|l|}{ HOSP4 } \\
\hline 2004 & Sim & 2.823 & 160 & 5,7 & 91 & 3,2 & 69 & 43,1 \\
\hline 2007 & & 2.734 & 128 & 4,7 & 124 & 4,5 & 4 & 3,1 \\
\hline \multicolumn{9}{|l|}{ HOSP6 } \\
\hline 2004 & Sim & 2.391 & 114 & 4,8 & 13 & 0,5 & 101 & 88,6 \\
\hline 2007 & & 2.564 & 137 & 5,4 & 26 & 1,0 & 111 & 81,0 \\
\hline \multicolumn{9}{|l|}{ HOSP7 } \\
\hline 2004 & Sim & 1.476 & 44 & 3,0 & 16 & 1,1 & 28 & 63,6 \\
\hline 2007 & & 1.463 & 23 & 1,6 & 8 & 0,5 & 15 & 65,2 \\
\hline \multicolumn{9}{|l|}{ HOSP8 } \\
\hline 2004 & Sim & 1.409 & 45 & 3,2 & 12 & 0,9 & 33 & 73,3 \\
\hline 2007 & & 1.011 & & & 43 & 4,3 & & \\
\hline
\end{tabular}

n.d.: não disponível.

Nota: os hospitais estão localizados nos seguintes municípios: HOSP1 em João Pessoa (Paraíba), HOSP2 em Joinville (Santa Catarina), HOSP3 e HOSP7 em Florianópolis (Santa Catarina), HOSP4 em Campinas (São Paulo), HOSP5 em Belo Horizonte (Minas Gerais), HOSP6 em Salvador (Bahia) e HOSP8 em São Paulo (São Paulo).

correio eletrônico, exceto a SMS de Florianópolis. Foram realizadas ações específicas em quatro hospitais, conduzidas por profissionais de cada estabelecimento em conjunto com a sua direção. Reuniões de capacitação em vigilância epidemiológica de anomalias congênitas foram realizadas em todos esses hospitais. Ações específicas no fluxo de informação ocorreram em dois deles, incluindo mudança de local e momento do preenchimento da Declaração de Nascido Vivo; o preenchimento passou a ser executado no alojamento conjunto ou unidade neonatal, em vez de no centro obstétrico. Outra intervenção realizada foi em relação ao profissional responsável pelo preenchimento da Declaração de Nascido Vivo: no HOSP6, o preenchimento foi restringido a três profissionais de saúde e, no HOSP8, a su- pervisão do preenchimento do campo referente a anomalias congênitas foi designada a um pediatra (Tabela 3).

As SMS de São Paulo e Campinas conduziram ações especificamente direcionadas ao campo 34. Ambas conduziram reuniões de capacitação em vigilância epidemiológica de anomalias congênitas nos principais hospitais de seus respectivos municípios. A SMS de São Paulo realizou atividades de capacitação com os todos os profissionais envolvidos na coleta, codificação e digitação da informação, assim como cursos sobre diagnóstico e codificação de anomalias congênitas e sobre a entrada da informação no banco de dados (Tabela 4).

As ações desenvolvidas pelo Ministério da Saúde para aprimorar o campo 34 incluíram a 
Comparação da validade da informação sobre anomalias congênitas no Sistema de Informações sobre Nascidos Vivos (SINASC) em 2004 e 2007.

\begin{tabular}{|c|c|c|c|c|c|}
\hline & $\begin{array}{c}\text { Ação de } \\
\text { aprimoramento } \\
\text { (campo 34) }\end{array}$ & $\begin{array}{l}\text { Sensibilidade } \\
\text { (IC95\%) }\end{array}$ & $\begin{array}{c}\text { Especificidade } \\
\text { (IC95\%) }\end{array}$ & $\begin{array}{c}\text { VPP } \\
\text { (IC95\%) }\end{array}$ & $\begin{array}{c}\text { VPN } \\
\text { (IC95\%) }\end{array}$ \\
\hline \multicolumn{6}{|l|}{ HOSP1 } \\
\hline 2004 & Não & $62,7(57,2-68,0)$ & $99,4(99,1-99,5)$ & $81,7(76,6-85,9)$ & $98,4(98,0-98,6)$ \\
\hline 2007 & & $41,7(36,4-47,0)$ & $99,0(98,7-99,3)$ & $71,3(64,9-77,6)$ & $96,6(96,2-97,1)$ \\
\hline \multicolumn{6}{|l|}{ HOSP2 } \\
\hline 2004 & Não & $50,5(40,8-60,2)$ & $99,5(99,3-99,7)$ & $63,7(53,8-72,6)$ & $99,2(98,9-99,4)$ \\
\hline 2007 & & $56,3(46,7-65,9)$ & $99,4(99,2-99,6)$ & $63,7(53,9-73,6)$ & $99,2(99,0-99,4)$ \\
\hline \multicolumn{6}{|l|}{ HOSP3 } \\
\hline 2004 & Não & $53,6(44,8-62,2)$ & $99,8(99,5-99,9)$ & $88,2(79,1-93,6)$ & $98,5(98,1-98,9)$ \\
\hline 2007 & & $56,5(46,4-66,7)$ & $99,5(99,3-99,7)$ & $74,3(64,0-84,5)$ & $98,9(98,6-99,2)$ \\
\hline \multicolumn{6}{|l|}{ HOSP5 } \\
\hline 2004 & Não & $66,5(60,0-72,6)$ & $99,3(98,9-99,6)$ & $89,9(84,2-93,5)$ & $97,2(96,5-97,8)$ \\
\hline 2007 & & $32,2(26,2-38,2)$ & $99,7(99,5-99,9)$ & $91,6(85,6-97,5)$ & $94,1(93,2-95,0)$ \\
\hline \multicolumn{6}{|l|}{ HOSP4 } \\
\hline 2004 & Sim & $56,9(49,1-64,4)$ & $99,9(99,6-99,9)$ & $96,8(90,7-98,9)$ & $97,5(96,9-98,1)$ \\
\hline 2007 & & $96,8(92,6-99,0)$ & $99,5(99,2-99,8)$ & $90,5(85,6-95,4)$ & $99,8(99,6-99,9)$ \\
\hline \multicolumn{6}{|l|}{ HOSP6 } \\
\hline 2004 & Sim & $11,4(6,5-18,3)$ & $99,9(99,7-100,0)$ & $92,9(63,3-99,0)$ & $95,7(94,9-96,6)$ \\
\hline 2007 & & $19,0(12,4-25,5)$ & $99,8(99,5-99,9)$ & $83,9(67,8-93,8)$ & $95,6(94,8-96,4)$ \\
\hline \multicolumn{6}{|l|}{ HOSP7 } \\
\hline 2004 & Sim & $36,4(23,2-51,2)$ & $99,7(99,3-99,9)$ & $80,0(57,6-91,8)$ & $98,1(97,4-98,8)$ \\
\hline 2007 & & $34,8(15,3-54,2)$ & $99,8(99,4-99,9)$ & $72,7(42,2-92,5)$ & $99,0(98,4-99,5)$ \\
\hline \multicolumn{6}{|l|}{ HOSP8 } \\
\hline 2004 & Sim & $26,7(15,3-40,9)$ & $100,0(99,8-100,0)$ & $100,0(100,0)$ & $97,6(96,8-98,4)$ \\
\hline 2007 & & n.d. & n.d. & n.d. & n.d. \\
\hline
\end{tabular}

IC95\%: intervalo de 95\% de confiança; n.d.: não disponível; VPN: valor preditivo negativo; VPP: valor preditivo positivo. Nota: os hospitais estão localizados nos seguintes municípios: HOSP1 em João Pessoa (Paraíba), HOSP2 em Joinville (Santa Catarina), HOSP3 e HOSP7 em Florianópolis (Santa Catarina), HOSP4 em Campinas (São Paulo), HOSP5 em Belo Horizonte (Minas Gerais), HOSP6 em Salvador (Bahia) e HOSP8 em São Paulo (São Paulo).

abertura do edital MCT-CNPq/MS-SCTIE-DECIT - no. 21/2006 em colaboração com o Ministério da Ciência e Tecnologia (MCT) e por intermédio do Conselho Nacional de Desenvolvimento Científico e Tecnológico (CNPq). O objetivo do edital foi fomentar a pesquisa sobre Genética Clínica, por meio do apoio a projetos de pesquisa que dessem subsídios para a formulação, implementação e avaliação de ações públicas voltadas para a Atenção em Genética Clínica no SUS. Dos 14 projetos aprovados no edital, um deles era relacionado à temática "Anomalias Congênitas", na linha de pesquisa "Avaliação da qualidade do preenchimento do campo 34 da Declaração de Nascido Vivo e ações que possam melhorar o instrumento". Os resultados desse projeto ainda não foram publicados.
Além disso, no início de 2007, o Ministério da Saúde realizou um seminário com gestores do SINASC de algumas secretarias estaduais de saúde (SES) e SMS, membros do ECLAMC e especialistas em codificação, para discutir iniciativas de aprimoramento do preenchimento do campo 34 na Declaração de Nascido Vivo. O Ministério da Saúde não determinou novos procedimentos, nem aprovou resoluções ou portarias em relação à notificação das anomalias congênitas pelo SINASC desde sua implementação no ano 1999.

\section{Discussão}

O SINASC apresentava, em 2004, baixa sensibilidade em relação à informação sobre anomalias 
Tabela 3

Intervenções realizadas pelos hospitais, 2005 a 2007

\begin{tabular}{|c|c|c|c|}
\hline Hospital & Município (Estado) & Período & Intervenções \\
\hline HOSP4 & Campinas (São Paulo) & Abril/2006 & $\begin{array}{l}\text { - Simpósio sobre anomalias congênitas e vigilância } \\
\text { epidemiológica em anomalias congênitas }\end{array}$ \\
\hline HOSP6 & Salvador (Bahia) & Fevereiro/2007 & $\begin{array}{c}\text { - Reunião com diretor da unidade neonatal } \\
\text { - Mudança do local de preenchimento da } \\
\text { Declaração de Nascido Vivo (do centro obstétrico } \\
\text { para o alojamento conjunto) } \\
\text { - Restrição no preenchimento da Declaração de } \\
\text { Nascido Vivo para três profissionais }\end{array}$ \\
\hline HOSP7 & Florianópolis (Santa Catarina) & 2005-2006 & $\begin{array}{l}\text { - Reunião com profissionais de saúde para } \\
\text { capacitação em notificação de anomalias congênitas }\end{array}$ \\
\hline HOSP8 & São Paulo (São Paulo) & Fevereiro/2007 & $\begin{array}{l}\text { - Reunião com profissionais de saúde para } \\
\text { capacitação em notificação de anomalias congênitas } \\
\text { - Mudança do local de preenchimento da } \\
\text { Declaração de Nascido Vivo (do centro obstétrico } \\
\text { para o alojamento conjunto) } \\
\text { - Mudança no dia de preenchimento da Declaração } \\
\text { de Nascido Vivo (de "ao nascimento" para próximo } \\
\text { à alta hospitalar) } \\
\text { - Supervisão, por pediatra, do preenchimento } \\
\text { da variável anomalia congênita da Declaração de } \\
\text { Nascido Vivo }\end{array}$ \\
\hline HOSP1 & João Pessoa (Paraíba) & - & - \\
\hline HOSP2 & Joinville (Santa Catarina) & - & - \\
\hline HOSP3 & Florianópolis (Santa Catarina) & - & - \\
\hline HOSP5 & Belo Horizonte (Minas Gerais) & - & - \\
\hline
\end{tabular}

congênitas, com, pelo menos, $40 \%$ de subnotificação dos casos de anomalias congênitas, tanto em anomalias maiores quanto nas menores. Esperava-se que essa situação melhorasse ao longo do tempo e que ações específicas visando a um aprimoramento na qualidade das informações sobre anomalias congênitas fossem realizadas pelos gestores desse sistema de informação. Todavia, pela análise, verifica-se que, após sete anos de implementação do campo sobre anomalias congênitas no SINASC, o perfil de notificação não melhorou significativamente entre os anos de 2004 e 2007. Em adição, poucas ações específicas ocorreram visando ao aperfeiçoamento desse campo.

Os resultados evidenciaram persistência da baixa sensibilidade na notificação das anomalias congênitas. Ao contrário do esperado, nos HOSP1 e HOSP5 houve maior subnotificação, com redução em mais de $40 \%$ na sensibilidade entre 2004 e 2007. Em quatro hospitais houve certa melhora na notificação, contudo a subnotificação ainda ocorre em, pelo menos, $40 \%$ dos casos. Apesar da sensibilidade baixa, o SINASC manteve elevada especificidade e elevados valores preditivos positivo e negativo.

Medidas para o aprimoramento da notificação dessas condições foram tomadas em poucos locais; somente duas SMS e quatro hospitais realizaram ações específicas. Em São Paulo, apesar de não ser possível avaliar a validade de diagnóstico no hospital (HOSP8), o aumento da prevalência em mais de $300 \%$ sugere que as ações de capacitação realizadas pelo hospital e pela SMS tiveram impacto significativo na notificação das anomalias congênitas. Ainda assim, com exceção deste município, as demais SMS e hospitais realizaram intervenções que não envolveram o principal fator necessário para mudanças nesse sistema de informação, ou seja, a capacitação de todos os profissionais envolvidos no processo do diagnóstico, preenchimento e codificação da Declaração de Nascido Vivo em relação ao diagnóstico de anomalias congênitas.

Em outros países, instrumentos semelhantes à Declaração de Nascido Vivo, como o "birth 
Intervenções realizadas pelas secretarias municipais de saúde (SMS), 2005 a 2007.

\begin{tabular}{|c|c|c|}
\hline Município (Estado) & Período & Intervenções \\
\hline \multirow[t]{4}{*}{ Campinas (São Paulo) } & Novembro/2005 & - Reunião com todos os hospitais do município na SMS \\
\hline & Dezembro/2005 a & - Reuniões em cada hospital \\
\hline & Fevereiro/2006 & \\
\hline & 2006-2007 & $\begin{array}{c}- \text { Dados do ECLAMC (CAISM) utilizados para completar o } \\
\text { banco de dados do SINASC }\end{array}$ \\
\hline \multirow[t]{7}{*}{ São Paulo (São Paulo) } & 2006-2007 & - Cursos de capacitação dos profissionais de saúde \\
\hline & & (enfermeiras e pediatras) em diagnóstico de \\
\hline & & anomalias congênitas, preenchimento do campo 34 \\
\hline & & e codificação de anomalias congênitas pela CID-10 \\
\hline & & - Reunião com os diretores clínicos dos hospitais do \\
\hline & & município \\
\hline & & - Capacitação dos digitadores do SINASC \\
\hline Belo Horizonte (Minas Gerais) & - & - \\
\hline João Pessoa (Paraíba) & - & - \\
\hline Joinville (Santa Catarina) & - & - \\
\hline Florianópolis (Santa Catarina) & - & - \\
\hline Salvador (Bahia) & - & - \\
\hline
\end{tabular}

CAISM: Centro de Atenção Integral à Saúde da Mulher; CID-10: Classificação Internacional de Doenças, 10a revisão; ECLAMC: Estudo Colaborativo Latino-Americano de Malformações Congênitas; SINASC: Sistema de Informações sobre Nascidos Vivos.

certificate", nos Estados Unidos, e "live-birth notification", na Europa, são utilizados como fonte de dados para programas de vigilância epidemiológica em anomalias congênitas. Entretanto, existem poucos estudos publicados que avaliam ações específicas para aperfeiçoar a notificação das anomalias congênitas no instrumento em questão. Pesquisas norte-americanas que avaliaram a introdução do uso de lista prédeterminada de anomalias congênitas no birth certificate não encontraram melhora significativa na sensibilidade para detectar anomalias congênitas 11,12,13. Minton et al. 14 relataram o aumento na sensibilidade do birth certificate na notificação de anomalias congênitas após três intervenções realizadas simultaneamente: a transferência da responsabilidade de notificar as anomalias congênitas do obstetra para o pediatra, a criação de um formulário especifico para anomalias congênitas no prontuário do recém-nascido e a designação de apenas um profissional capacitado, por hospital, para revisão do prontuário e preenchimento dessa variável no birth certificate. Por sua vez, Hemminki et al. 15 referem piora na notificação das anomalias congênitas na Finlândia após a criação de formulários específicos e da transferência de responsabilidade do preenchimento de técni- cos administrativos para médicos. Os autores do estudo finlandês sugerem que o formulário adicional aumentou a carga de trabalho dos médicos; além disso, estes profissionais tendem a julgar clinicamente quais anomalias deveriam ser ou não notificadas, o que levou a uma diminuição da notificação.

Esses estudos mostram que as mesmas intervenções podem ter efeitos diversos, dependendo do local, e destacam o papel da capacitação do profissional envolvido no processo de notificação para o aprimoramento desse procedimento.

A qualidade dos dados em vigilância epidemiológica é influenciada principalmente pela clareza dos formulários, pela qualidade da capacitação e supervisão dos profissionais que preenchem esses formulários e pelo cuidado no manejo dos dados 1. A Declaração de Nascido Vivo é clara na sua pergunta: "Detectada alguma malformação congênita elou anomalia cromossômica?"; ademais, a pergunta aberta, sem um grupo de anomalias previamente determinadas, permite a notificação de qualquer tipo de condição, evitando que anomalias pouco comuns não sejam notificadas. Todavia, o manual de preenchimento da Declaração de Nascido Vivo 16 não estabelece nenhuma definição de "malformação congênita”. Os responsáveis pelo preenchimento 
podem ter dúvidas se, por exemplo, anomalias menores ou erros inatos do metabolismo devem ser incluídos, o que pode levar à subnotificação. O Ministério da Saúde deve definir, no manual, o que é considerado "malformação congênita", determinando, por exemplo, que todas as anormalidades devem ser notificadas, tanto maiores quanto menores; deve, ainda, especificar normas para diagnósticos que geralmente geram dúvidas, como, por exemplo, quando considerar criptorquidia ou persistência do canal arterial como uma anomalia congênita.

Outra questão é em relação à capacitação dos profissionais, tanto dos médicos responsáveis pelo diagnóstico e descrição das anomalias, como dos responsáveis pela transcrição desse diagnóstico para a Declaração de Nascido Vivo (realizado pelo próprio médico ou por outro profissional). As anomalias congênitas estão presentes em aproximadamente $2 \%$ dos nascidos vivos, porém essa proporção reúne vários tipos distintos de anomalias, com diferentes níveis de gravidade, fazendo com que, individualmente, cada anomalia congênita seja um evento raro, implicando dificuldade na capacitação do seu reconhecimento e descrição 17,18 . Os médicos nem sempre estão capacitados para o diagnóstico clínico e, principalmente, para a descrição dessas condições. Some-se a isso o fato de que os responsáveis pelo preenchimento não são capacitados para reconhecer esses casos na revisão do prontuário, para que, assim, possam também fazer a notificação.

Nesse sentido, os cursos de capacitação e a publicação, em 2008, do Manual de Anomalias Congênitas 19 pela SMS-SP são iniciativas que poderiam ser adotadas por outras secretarias municipais de saúde. Assim, o preenchimento da Declaração de Nascido Vivo poderia ser feito por qualquer profissional treinado, desde que este pudesse obter os dados necessários no prontuário. A capacitação do médico na descrição das anomalias congênitas e a criação de um espaço padronizado no prontuário reservado para o diagnóstico dessa morbidade tornaria o diagnóstico (ou ausência de) mais acessível para o preenchimento adequado do campo 34 da Declaração de Nascido Vivo.

Entre as limitações do presente estudo, podese mencionar que a análise foi realizada somente em hospitais de ensino da rede do SUS, isto é, os resultados aqui observados têm abrangência localizada, não sendo, a priori, generalizáveis para o conjunto da rede de atenção pública do SUS. Não se sabe ainda qual a qualidade da informação referente às anomalias congênitas na Declaração de Nascido Vivo em outros locais, mas estima-se, com base no Departamento de
Informática do SUS (DATASUS), a existência de subnotificação intensa em todas as regiões do Brasil. Os hospitais selecionados, por serem de ensino, corresponderiam a um dos melhores cenários na notificação das anomalias congênitas, o que, se verdadeiro, seria indicativo de que o SINASC demanda um grau de aprimoramento para ser usado como fonte para a determinação da prevalência dessas morbidades no Brasil.

Além disso, no HOSP4, em Campinas, não foi possível determinar se a melhora na sensibilidade ocorreu pelo compartilhamento do banco de dados do ECLAMC com o SINASC, ou se houve melhora na notificação das anomalias congênitas na Declaração de Nascido Vivo pela equipe do hospital. A complementação de dados obtida pela vinculação dos bancos de dados do SINASC e ECLAMC em Campinas caminha na desejada direção da vigilância epidemiológica com múltiplas fontes de dados, contudo o ECLAMC é um projeto de pesquisa colaborativo nãoinstitucional e, portanto, a participação de um hospital não é permanente. Outrossim, o compartilhamento do banco de dados não modifica a coleta da informação no hospital, logo devem ser priorizadas ações no hospital que capacitem os profissionais responsáveis pelo diagnóstico e pelo preenchimento da Declaração de Nascido Vivo em relação às anomalias congênitas.

No estudo realizado em 2004, identificamos, além da baixa sensibilidade, baixa confiabilidade da codificação dessas condições 9 . A codificação não foi o objetivo do presente estudo, mas, considerando que não foram realizadas ações específicas de capacitação dos profissionais das SMS para codificação das anomalias congênitas, acreditamos que a confiabilidade da codificação permanece baixa.

Aproximadamente três milhões de nascimentos ocorrem ao ano no Brasil (DATASUS. http:// www.datasus.gov.br, acessado em 20/Mar/2009) e estima-se que ao menos 60 mil recém-nascidos são portadores de anomalias congênitas. A caracterização dessas crianças, da prevalência dos diferentes tipos de anomalias congênitas e a distribuição destas pelo território nacional são informações de importância para o planejamento e implementação de programas que atendam as demandas dos portadores dessas condições e de suas famílias. Portanto, a criação, pelo Ministério da Saúde, de um programa nacional de vigilância epidemiológica em anomalias congênitas capaz de fornecer esses indicadores de forma acurada é uma necessidade para o planejamento de políticas de saúde, principalmente para a saúde infantil. A base de dados do SINASC constitui a fonte principal de dados para o desenvolvimento de tal programa. 
Este projeto avaliou a qualidade dos dados sobre anomalias congênitas no SINASC em dois períodos, 2004 e 2007. Conclui-se que esse sistema de informação em saúde apresenta baixa sensibilidade, quantificada neste estudo, mas da qual já se suspeitava previamente, pelas baixas prevalências reportadas pelo SINASC, em comparação às prevalências esperadas. Ainda assim, a expectativa de que ações seriam tomadas pelos gestores de saúde para a melhoria da qualidade desse campo não se realizou. A falta de mecanismos de controle da qualidade dessa informação no hospital e na SMS, aliada à ausência de divulgação sobre a importância da vigilância epidemiológica em anomalias congênitas perpetuam a subnotificação, comprometendo a qualidade desses dados. Os resultados apontam para a necessidade urgente de se investir na qualidade da informação, seja no preenchimento da Declaração de Nascido Vivo, seja na codificação, como medida indispensável para avançar no compromisso com a melhoria da qualidade do SINASC e na implementação de um programa nacional de vigilância epidemiológica em anomalias congênitas.

\section{Resumo}

Este estudo comparou a validade dos diagnósticos de anomalias congênitas do Sistema de Informações sobre Nascidos Vivos (SINASC), em oito hospitais distribuídos em sete municípios do Brasil, totalizando 27.945 nascidos vivos em 2004 e 25.905 em 2007. Além disso, descreveu ações específicas realizadas para o aprimoramento da qualidade dos dados desse campo. Para a análise da validade, foi utilizado o Estudo Colaborativo Latino-Americano de Malformações Congênitas (ECLAMC) como padrão-ouro. Em 2004, pelo menos $40 \%$ dos casos de anomalias congênitas eram subnotificados, situação que não foi modificada em 2007. Observou-se aumento significativo na sensibilidade somente em um hospital, de $56,9 \%$ para $96,8 \%$. Em dois hospitais, houve diminuição significativa na sensibilidade, de 62,7\% para 41,7\% e de 66,5\% para $32,2 \%$. Os valores preditivos positivo e negativo e especificidade permaneceram acima de $80 \%$. Apenas duas secretarias municipais de saúde e quatro hospitais fizeram algum tipo de ação específica para a melhoria do campo 34. Os resultados apontam para a necessidade de se investir na qualidade da informação sobre anomalias congênitas no SINASC.

Anormalidades Congênitas; Declaração de Nascimento; Sistemas de Informação

\section{Colaboradores}

D. V. Luquetti participou da análise e interpretação dos dados, organização e redação do artigo, revisão e edição final. R. J. Koifman participou da organização e redação do artigo e revisão final.

\section{Agradecimentos}

Agradecemos à coordenação do Estudo Colaborativo Latino-Americano de Malformações Congênitas (ECLAMC), especialmente ao Dr. Eduardo E. Castilla e à Dra. Maria da Graça Dutra, pela permissão e apoio para consulta aos bancos de dados; à Fundação de Amparo à Pesquisa do Estado do Rio de Janeiro (FAPERJ), pelo apoio a D. V. Luquetti; ao Conselho Nacional de Desenvolvimento Científico e Tecnológico (CNPq) e à FAPERJ, pelo apoio a R.J. Koifman. 


\section{Referências}

1. Brookmeyer R, Stroup DF. Monitoring the health of populations: statistical principles and methods for public health surveillance. Oxford: Oxford University Press; 2004.

2. Geremias AL, Almeida MF, Flores LPO. Avaliação das declarações de nascido vivo como fonte de informação sobre defeitos congênitos. Rev Bras Epidemiol 2009: 12:60-8

3. Almeida MF, Alencar GP, França Jr. I, Novaes HMD Siqueira AAF, Schoeps D, et al. Validade das informações das declarações de nascidos vivos com base em estudo de caso-controle. Cad Saúde Pública 2006; 22:643-52.

4. Guerra FA, Llerena Jr. JC, Gama SGN, Cunha CB, Theme Filha MM. Defeitos congênitos no Município do Rio de Janeiro, Brasil: uma avaliação através do SINASC (2000-2004). Cad Saúde Pública 2008; 24:140-9.

5. Righeto ALC, Huber J, Machado JC, Melo DG. Anomalias congênitas: validade das informações das declarações de nascido vivo em uma maternidade de Ribeirão Preto, São Paulo. Pediatria (São Paulo) 2008; 30:159-64.

6. Theme Filha MM, Gama SGN, Cunha CB, Leal MC. Confiabilidade do Sistema de Informações sobre Nascidos Vivos Hospitalares no Município do Rio de Janeiro, 1999-2001. Cad Saúde Pública 2004; 20 Suppl 1:S83-S91.

7. Guerra FAR, Llerena Jr. JC, Gama SGN, Cunha CB, Theme Filha MM. Confiabilidade das informações das declarações de nascido vivo com registro de defeitos congênitos no Município do Rio de Janeiro, Brasil, 2004. Cad Saúde Pública 2008; 24: 438-46.

8. Luquetti DV, Koifman RJ. Quality of reporting on birth defects in birth certificates: case study from a Brazilian reference hospital. Cad Saúde Pública 2009; 25:1721-31.
9. Luquetti DV, Koifman RJ. Validity and reliability of the Brazilian birth certificate for birth defects data in 2004. J Registry Manag; no prelo.

10. Castilla EE, Orioli IM. ECLAMC: the Latin-American collaborative study of congenital malformations. Community Genet 2004; 7:76-94.

11. Watkins ML, Edmonds L, McClearn A, Mullins L Mulinare J, Khoury M. The surveillance of birth defects: the usefulness of the revised US standard birth certificate. Am J Public Health 1996; 86:731-4.

12. Piper JM, Mitchel Jr. EF, Snowden M, Hall C, Adams M, Taylor P. Validation of 1989 Tennessee birth certificates using maternal and newborn hospital records. Am J Epidemiol 1993; 137:758-68.

13. Frost F, Starzyk P, George S, McLaughlin JF. Birth complication reporting: the effect of birth certificate design. Am J Public Health 1984; 74:505-6.

14. Minton SD, Seegmiller RE. An improved system for reporting congenital malformations. JAMA 1986; 256:2976-9.

15. Hemminki E, Merilainen J, Teperi J. Reporting of malformations in routine health registers. Teratology 1993; 48:227-31.

16. Fundação Nacional de Saúde. Manual de instruções para o preenchimento da Declaração de Nascido Vivo. Brasília: Ministério da Saúde; 2001.

17. Mastroiacovo P. Malformazioni congenite: epidemiologia e prevenzione. Prospettive in Pediatria 2008; 38:59-72.

18. Dastgiri S, Stone DH, Le Ha C, Gilmour WH. Prevalence and secular trend of congenital anomalies in Glasgow, UK. Arch Dis Child 2002; 86:257-63.

19. Coordenação de Epidemiologia e Informação, Secretaria Municipal da Saúde. Declaração de Nascido Vivo: campo 34 - manual de anomalias congênitas. São Paulo: Secretaria Municipal da Saúde; 2008.

Recebido em 10/Jan/2010

Versão final reapresentada em 15/Jun/2010

Aprovado em 20/Jul/2010 